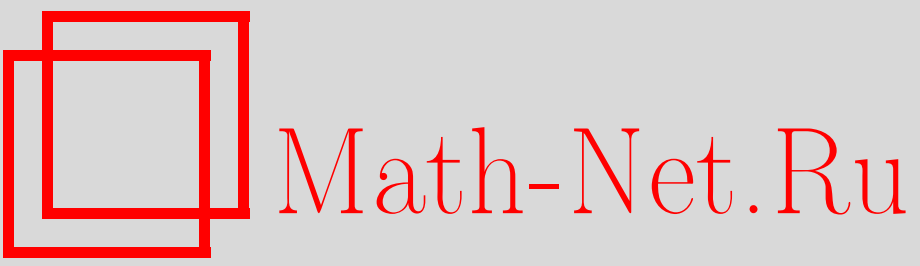

А. А. Бейлинсон, Структура фундаментального решения уравнения Дирака, TMФ, 2005, том 145, номер 2, 157-164

DOI: https://doi.org/10.4213/tmf1891

Использование Общероссийского математического портала Math-Net.Ru подразумевает, что вы прочитали и согласны с пользовательским соглашением

http://www.mathnet.ru/rus/agreement

Параметры загрузки:

IP: 34.229 .45 .116

26 апреля 2023 г., 12:39:56 


\section{СТРУКТУРА ФУНДАМЕНТАЛЬНОГО РЕШЕНИЯ УРАВНЕНИЯ ДИРАКА}

Показано, что евклидова мера Дирака как обобщенная мера счетно-аддитивна.

Ключевые слова: счетно-аддитивная евклидова мера Дирака электрона, нейтрино.

Напомним, что уравнение Дирака

$$
i \gamma^{\mu} \frac{\partial}{\partial x^{\mu}}-m I=0
$$

имеет фундаментальное решение

$$
D_{t}^{m}(x)=\gamma^{0}\left(\gamma^{\mu} \frac{\partial}{\partial x^{\mu}}-i m I\right) G_{t}(x)
$$

где $G_{t}(x)$ - функция Паули-Йордана,

$$
G_{t}(x)=\frac{\delta\left(t^{2}-r^{2}\right)}{2 \pi}-\frac{m}{4 \pi} \theta(t-r) \frac{J_{1}\left(m \sqrt{t^{2}-r^{2}}\right)}{\sqrt{t^{2}-r^{2}}} .
$$

Здесь $\gamma^{\mu}$ - матрицы Дирака $\left(\gamma^{\mu} \gamma^{\nu}+\gamma^{\nu} \gamma^{\mu}=2 g^{\mu \nu}, \mu, \nu=0,1,2,3, g^{\mu \nu}\right.$ - метрический тензор [1], $\left.x^{\mu}=\left\{x^{0}=t, x\right\}, x=\left\{x^{1}, x^{2}, x^{3}\right\}, r=|x|\right), m$ - масса электрона, а $\theta$ и $J_{1}$ - функции Хевисайда и Бесселя, соответственно. Мы пользуемся системой единиц, в которой скорость света и постоянная Планка равны единице.

Таким образом, фундаментальное решение представляет собой матричнозначную обобшенную функцию от пространственных переменных $x$ на пространстве $Z$ целых аналитических основных функций $\psi(x)$ [2]; время $t$ будем считать параметром.

Так как $D_{t}^{m}(x)$ является финитным функционалом, легко можно найти его образ Фурье

$$
\widetilde{D}_{t}^{m}(\omega)=I \cos \left(t \sqrt{m^{2}+\rho^{2}}\right)-i \gamma^{0} \frac{(\gamma, \omega)+m I}{\sqrt{m^{2}+\rho^{2}}} \sin \left(t \sqrt{m^{2}+\rho^{2}}\right),
$$

где $\rho$ - модуль вектора $\omega, \omega=\left\{\omega_{1}, \omega_{2}, \omega_{3}\right\},(\gamma, \omega)=\sum_{k=1}^{3} \gamma^{k} \omega_{k}$.

\footnotetext{
* Российский университет дружбы народов, Москва, Россия. E-mail: alal@m9com.ru
} 
Используя известные свойства $\gamma$-матриц Дирака, выражение (3) можно привести к виду

$$
\widetilde{D}_{t}^{m}(\omega)=e^{-i \gamma^{0}[(\gamma, \omega)+m I] t}
$$

(формула Эйлера), который также можно получить непосредственно, записывая в тригонометрическом базисе фундаментальное решение уравнения Дирака (1).

Ясно, что (4) является обобщенной функцией на пространстве $K$ основных функций [2]. Вместе с тем она аналитична по $t$, так что можно перейти к ее евклидову аналогу

$$
\widetilde{D}_{-i t}^{m}(\omega)=e^{t \gamma^{0}[(\gamma, \omega)+m I]}
$$

Рассмотрим сначала случай $m=0$ (нейтрино). При этом

$$
\widetilde{D}_{-i t}^{0}(\omega)=e^{t \gamma^{0}(\gamma, \omega)}
$$

Так как матрица в показателе экспоненты эрмитова, ее можно привести к диагональному виду. Легко проверить, что это осуществляется эрмитовым (и унитарным) оператором, не зависяшим от времени,

$$
\widetilde{T}=\widetilde{T}^{-1}=\frac{1}{\sqrt{2}}\left[\gamma^{0}\left(\gamma, \omega_{e}\right)+\gamma^{0}\right],
$$

где $\omega_{e}$ - единичный вектор, направленный вдоль $\omega$.

В результате получаем

$$
t \gamma^{0}(\gamma, \omega)=\widetilde{T} t \gamma^{0} \rho \widetilde{T}
$$

так что

$$
\widetilde{D}_{-i t}^{0}(\omega)=\widetilde{T} e^{t \gamma^{0} \rho} \widetilde{T}
$$

Рассмотрим подробнее экспоненциал, возникший в последней формуле:

$$
\widetilde{B}_{-i t}(\omega)=e^{t \gamma^{0} \rho}
$$

Это выражение является образом Фурье некоторой матричной причинной функции $B_{-i t}(x)$. Ее свойства однозначно определяют свойства причинной функции Грина нейтрино $D_{-i t}^{0}(x)$, которая представляет собой свертку трех функций: прообразов $\widetilde{T}$, $\widetilde{B}_{-i t}(\omega)$ и $\widetilde{T}$.

Отметим при этом, что прообраз $\widetilde{T}(\omega)$ легко может быть найден, так как $\widetilde{T}$ - однородная функция от $\omega[2]$. Если обозначить этот прообраз $T(x)$, то, очевидно,

$$
\int D_{-i t}^{0}(x) \psi(x) d x=\int T(x) B_{-i t}(y) T(z) \psi(x+y+z) d x d y d z
$$


Поскольку $D_{-i t}^{0}(x), B_{-i t}(x)$ - причинные функции, справедливо равенство

$$
\begin{aligned}
& \int\left[\prod_{k=1}^{l} D_{-i \Delta t_{k}}^{0}\left(\Delta x_{k}\right)\right] \psi\left(x_{l}\right) d x_{1} \ldots d x_{l}= \\
& \quad=\int T(x)\left[\prod_{k=1}^{l} B_{-i \Delta t_{k}}\left(\Delta x_{k}\right)\right] T(z) \psi\left(x+x_{l}+z\right) d x d x_{1} \ldots d x_{l} d z
\end{aligned}
$$

(напомним, что $\sum_{k=1}^{l} \Delta x_{k}=x_{l}$, кроме того, здесь мы положили $x_{0}=0$ ).

Поэтому такие важнейшие аналитические свойства предмер, отвечающих $D_{-i t}^{0}(x)$ и $B_{-i t}(x)$, как, например, счетная аддитивность, имеют место одновременно.

Отметим также, что так как $\gamma^{0}$ - диагональная матрица, $\widetilde{B}_{-i t}(\omega)$ также диагональна, отчего уравнение (10) распадается на четыре отдельные независимые задачи, попарно одинаковые, так что возникают два образа Фурье

$$
\tilde{b}_{-i t}(\omega)=e^{-t \rho}, \quad \tilde{b}_{-i t}^{*}=e^{t \rho} .
$$

Ясно, что первая из них является положительно определенной непрерывной обобщенной функцией и удовлетворяет условиям теоремы Бохнера [3], так что $b_{-i t}(x)$ - положительная и в данном случае нормированная функция. Найдем ее.

Напомним, что $\tilde{b}_{-i t}(\omega)$ является обобшенной функцией на пространстве $K$ основных функций, и решение задачи поиска $b_{-i t}(\omega)$ сводится к обратному преобразованию Фурье функции $e^{-t \rho}$. Эта функция абсолютно интегрируема, и ее обратное преобразование Фурье известно [4]:

$$
b_{-i t}(x)=\frac{\pi^{-2} t}{\left(t^{2}+r^{2}\right)^{2}} .
$$

Так как функционал $b_{-i t}(x)$ является аналитическим в $Z$ и в то же время аналитическим по параметру $t$, поэтому $b_{-i t}^{*}(x)=b_{i t}(x)$ как аналитическое продолжение на комплексную плоскость $t$ функционала (14) сушествует и единствен.

Воспользуемся этим важным обстоятельством для получения ответа на вопрос о том, обладает ли обобшенная евклидова мера, отвечающая $b_{-i t}^{*}(x)$, одновременно с $b_{-i t}(x)$ свойством счетной аддитивности, имея в виду следуюшую возможность: при решении части задачи об эволюции, отвечающей $b_{-i t}^{*}(x)$, сначала аналитическим продолжением перейти к $b_{-i t}(x)$, для этой функции решить эволюционную задачу, после чего вновь вернуться к $b_{-i t}^{*}(x)$, используя аналитичность по времени. При этом мы обходим трудность, связанную с комплексностью $b_{-i t}^{*}(x)$, так же, как это происходит при переходе от уравнения Шредингера к уравнению Блоха в нерелятивистской квантовой механике.

В частности, в одномерном случае, прообразом $\tilde{b}_{-i t}^{*}\left(\omega_{1}\right)=e^{t\left|\omega_{1}\right|}$ является аналитический функционал

$$
b_{-i t}^{*}\left(x_{1}\right)=-\frac{t}{\pi\left(t^{2}+x_{1}^{2}\right)}+\delta\left(x_{1}+i t\right)+\delta\left(x_{1}-i t\right),
$$


в то время как прообразом $\tilde{b}_{-i t}\left(\omega_{1}\right)=e^{-t\left|\omega_{1}\right|}$ является функционал

$$
b_{-i t}\left(x_{1}\right)=\frac{t}{\pi\left(t^{2}+x_{1}^{2}\right)},
$$

так что $b_{-i t}^{*}\left(x_{1}\right)$ есть результат аналитического продолжения по времени $b_{-i t}\left(x_{1}\right)$ с положительной части действительной оси на отрицательную.

Ясно, что причинная функция (14) является сферически симметричной и, очевидно, негауссовой плотностью вероятности перехода в трехмерном пространстве.

Поэтому $b_{-i t}(x) \rightarrow \delta(x)$ при $t \rightarrow 0$ и, так как $b_{-i t}(x)-$ функционал типа функции, выполняется уравнение Эйнштейна-Смолуховского

$$
b_{-i t}(x)=\int b_{-i s}(y) b_{-i(t-s)}(x-y) d y, \quad 0 \leqslant s \leqslant t .
$$

Отметим, что гауссова плотность вероятности возникает при предельном переходе к нерелятивистскому уравнению Шредингера, как это будет показано в конце статьи.

Покажем теперь, как задание причинной функции $b_{-i t}(x)$ определяет вероятностную меру цилиндрического множества с элементами $a_{1}(\tau), \ldots, a_{n}(\tau)$, представляюшими собой заданные вектор-функции от времени, и $n$-мерным основанием. Иначе говоря, найдем вероятностную меру значений $A_{1}, \ldots, A_{n}$ линейных функционалов

$$
\int_{0}^{t}\left(a_{j}(\tau), d x(\tau)\right)=A_{j}, \quad j=1, \ldots, n .
$$

Для этого выделим $k$ последовательных моментов времени $t_{1}, \ldots, t_{k}=t$ и запишем равенство (15) в приближенном виде

$$
\sum_{l=1}^{k}\left(a_{j l}, \Delta x_{l}\right)=A_{j}
$$

Принцип причинности, которому удовлетворяет $b_{-i t}(x)$ как функционал, дает

$$
\int\left[\prod_{l=1}^{k} b_{-i \Delta t_{l}}\left(\Delta x_{l}\right)\right] \Psi\left(\Delta x_{1}+\cdots+\Delta x_{k}\right) d x_{1} \ldots d x_{k}=\int b_{-i t}(x) d x
$$

Рассмотрим теперь интеграл

$$
\int\left[\prod_{l=1}^{k} b_{-i \Delta t_{l}}\left(\Delta x_{l}\right)\right] \Psi\left(\sum_{l=1}^{k}\left(a_{1_{l}}, \Delta x_{l}\right), \ldots, \sum_{l=1}^{k}\left(a_{n_{l}}, \Delta x_{l}\right)\right) d x_{1} \ldots d x_{k}
$$

где $\Psi\left(A_{1}, \ldots, A_{n}\right) \in Z^{n}$. Такой интеграл, очевидно, сушествует для любых таких $\Psi$. 
Запишем теперь эту аналитическую целую функцию от $n$ переменных через интеграл Фурье:

$$
\begin{aligned}
& \Psi\left(\sum_{l=1}^{k}\left(a_{1_{l}}, \Delta x_{l}\right), \ldots, \sum_{l=1}^{k}\left(a_{n_{l}}, \Delta x_{l}\right)\right)= \\
& \quad=(2 \pi)^{-3 n} \int \exp \left\{-i \sum_{j=1}^{n} \sum_{l=1}^{k}\left(a_{j_{l}}, \Omega_{l} \Delta x_{l}\right)\right\} \Phi\left(\Omega_{1}, \ldots, \Omega_{n}\right) d \Omega_{1} \ldots d \Omega_{n}
\end{aligned}
$$

Здесь $\Phi\left(\Omega_{1}, \ldots, \Omega_{n}\right) \in K^{n}$.

Подставим это разложение в (17) и применим далее стандартные методы получения образа Фурье обобщенной функции [1]

$$
\int \prod_{l=1}^{k}\left[\int b_{-i \Delta t_{l}}\left(\Delta x_{l}\right) \exp \left\{i \Omega_{l} \sum_{j=1}^{n}\left(a_{j_{l}} \Delta x_{l}\right)\right\} d x_{l}\right]^{*} \Phi\left(\Omega_{1}, \ldots, \Omega_{n}\right) d \Omega_{1} \ldots d \Omega_{n}
$$

где * означает комплексное сопряжение.

Внутренний интеграл здесь известен, последнее выражение можно переписать как

$$
\int\left[\prod_{l=1}^{k} \tilde{b}_{-i \Delta t_{l}} \sum_{j=1}^{n} a_{j_{l}} \Omega_{j}\right] \Phi\left(\Omega_{1}, \ldots, \Omega_{n}\right) d \Omega_{1} \ldots d \Omega_{n}
$$

или, вспоминая (13), как

$$
\int \exp \left\{-\sum_{l=1}^{k} \Delta t_{l} \sqrt{\left(\sum_{j=1}^{n} \Omega_{j} a_{j_{l}}, \sum_{m=1}^{n} \Omega_{m} a_{m_{l}}\right)}\right\} \Phi\left(\Omega_{1}, \ldots, \Omega_{n}\right) d \Omega_{1} \ldots d \Omega_{n}
$$

Устремляя все $\Delta t_{j}$ к нулю, получаем образ Фурье распределения значений функционалов (15):

$$
\exp \left\{-\int_{0}^{t} \sqrt{\sum_{j, m=1}^{n} \Omega_{j} \Omega_{m}\left(a_{j}(\tau), a_{m}(\tau)\right)} d \tau\right\} .
$$

Понятно, что вектор-функции $a_{j}(\tau)$ должны быть такими, чтобы существовал интеграл в показателе экспоненты.

Легко проверить, что непрерывная обобшенная функция (18) положительно определена и поэтому по теореме Бохнера [3] определяет положительную согласованную меру цилиндрических множеств как обобшенную функцию на пространстве $S$ быстроубываюших функций, причем в данном случае эта мера нормирована. Согласно теореме Минлоса [3] эта мера может быть продолжена на борелевское тело множеств (счетно-аддитивна).

Согласно сказанному выше, свойством счетной аддитивности, как и функционалы, аналитические по $t$, обладают также мера $b_{-i t}(x)$ и мера $D_{-i t}^{0}(x)$, отвечающая нейтриHо. 
Вернемся к решению уравнения Дирака для электрона. В отличие от нейтрино, решение $D_{t}^{m}(x)$ не нормировано, как это следует из (5). Имея намерение установить абсолютную непрерывность мер, отвечающих этим задачам, укажем модифицированное уравнение Дирака для электрона, обладаюшее свойством нормированности. Для этого вспомним, что два разных решения уравнения Дирака $E_{t}(x)$ и $F_{t}(x)$ удовлетворяют уравнению непрерывности [1]

$$
\frac{\partial}{\partial x^{\mu}}\left(\dddot{E} \gamma^{\mu} F\right)=0
$$

где $E^{*}$ означает эрмитово сопряжение к $E$, а $\dddot{E}=E^{*} \gamma^{0}-$ сопряжение по Дираку.

Введем обозначение

$$
\Xi_{t}(x)=E_{t}^{*}(x) F_{t}(x)
$$

Если предположить, что $E_{t}(x)$ - фиксированное решение уравнения Дирака, то получившееся из (19) уравнение для $\Xi_{t}(x)$ будет, очевидно, эквивалентно уравнению Дирака для $F_{t}(x)$

Легко видеть, что

$$
E_{t}(x)=e^{-i \gamma^{0} m t}
$$

действительно есть решение уравнения Дирака $(1)$; тогда для $\Xi_{t}(x)$ с использованием свойств $\gamma$-матриц из (19) получается уравнение

$$
\frac{\partial \Xi}{\partial t}+\gamma^{0} e^{2 i \gamma^{0} m t}(\gamma, \nabla) \Xi=0
$$

Это уравнение, как уже отмечалось, эквивалентно уравнению Дирака для электрона, однако оно имеет структуру уравнения непрерывности, и потому его решение нормировано.

Пусть $\Xi_{t}(x)$ - фундаментальное решение уравнения (22), тогда его образ Фурье есть

$$
\widetilde{\Xi}_{t}(\omega)=\exp \left\{i \gamma^{0}(\gamma, \omega) \int_{0}^{t} e^{2 i \gamma^{0} m \tau} d \tau\right\} .
$$

Евклидов вариант этой функции имеет следующий вид:

$$
\widetilde{\Xi}_{-i t}(\omega)=\exp \left\{\gamma^{0}(\gamma, \omega) \int_{0}^{t} e^{2 \gamma^{0} m \tau} d \tau\right\}
$$

Отметим, что, как и в (23), здесь снова возникло хронологическое произведение. Сравним это выражение с образом Фурье фундаментального решения уравнения Дирака для нейтрино в евклидовом варианте (6).

Как было показано выше, образ Фурье меры цилиндрического множества

$$
\int_{0}^{t} e^{2 m \tau} d x(\tau)=A
$$


отвечаюший $b_{-i t}(x)$, где $A$ - фиксированный трехмерный вектор, есть

$$
\exp \left\{-\int_{0}^{t} \rho e^{2 m \tau} d \tau\right\}
$$

а образ Фурье меры того же цилиндрического множества, отвечающий $b_{-i t}^{*}(x)$, есть

$$
\exp \left\{\int_{0}^{t} \rho e^{2 m \tau} d \tau\right\}
$$

Отсюда, вспоминая (6), получаем

$$
\widetilde{D}_{-i G}^{0}(\omega)=e^{\gamma^{0}(\gamma, \omega) G}
$$

где $G=\int_{0}^{t} e^{2 \gamma^{0} m \tau} d \tau$. Сравнивая (24) и (26), окончательно имеем

$$
\widetilde{\Xi}_{-i t}(\omega)=\widetilde{D}_{-i G}^{0}(\omega) \text {. }
$$

Это означает, что переход от нейтрино (антинейтрино) к электрону (позитрону) сводится к подсчету плотности нейтринной (антинейтринной) евклидовой меры Дирака значений линейного функционала (25), где

$$
A=z_{t}-z_{0}, \quad a(\tau)=e^{ \pm 2 m \tau},
$$

причем $z_{t}-z_{0}$ интерпретируется как сдвиг координат электрона (позитрона).

Иначе говоря, фундаментальное решение уравнения (22) для евклидова электрона оказывается плотностью индуцированной евклидовой меры нейтрино функционала

$$
\int_{0}^{t} e^{2 \gamma^{0} m \tau} d x(\tau)=I\left(z_{t}-z_{0}\right) .
$$

При этом в силу структуры матрицы $\gamma^{0}$ в показателе экспоненты автоматически учитывается изменение знака времени при переходе от электрона к позитрону.

Поэтому евклидовы меры Дирака нейтрино $D_{-i t}^{0}(x)$ и электрона $D_{-i t}^{m}(x)$ счетноаддитивны и абсолютно непрерывны друг по другу.

В заключение покажем, как из фундаментального решения уравнения Дирака для электрона в евклидовом варианте, которое, как было показано, имеет негауссову структуру, получается при $C \rightarrow \infty(C$ - скорость света) фундаментальное решение диффузионного уравнения. Это проще всего обнаружить, пользуясь образами Фурье.

Вспомним, что образ Фурье фундаментального решения уравнения Дирака для электрона в евклидовом варианте дается формулой (4):

$$
\widetilde{D}_{-i t}^{m}(\omega)=e^{t \gamma^{0}[(\gamma, \omega)+m I]}
$$

где теперь $t$ нужно заменить на $C t$, причем считать $t$ комплексным, а $m-$ на $m C / \hbar(\hbar-$ постоянная Планка). 
Перепишем показатель экспоненты в виде

$$
\frac{t \gamma^{0}\left[\frac{(\gamma, \omega)}{C}+I \frac{m}{\hbar}\right] C^{2}}{\sqrt{\left(\frac{m C}{\hbar}\right)^{2}+\rho^{2}}} \frac{m C}{\hbar} \sqrt{1+\left(\frac{\rho \hbar}{m C}\right)^{2}},
$$

или в виде

$$
t \gamma^{0}\left[\rho^{2} \frac{\hbar}{2 m}+\frac{m C^{2}}{\hbar}\right]
$$

в пределе больших $C$.

Учет первого слагаемого в показателе экспоненты (напомним, что обобшенные функции $\tilde{b}_{-i t}^{*}(\omega)$ и $\tilde{b}_{-i t}(\omega)$ являются единым аналитическим по $t$ функционалом) точно дает при мнимом $t$ образ Фурье фундаментального решения уравнения Шредингера, а при действительном отрицательном $t$ - образ Фурье фундаментального решения уравнения диффузии. Второе слагаемое имеет структуру, всегда возникающую при переходе к нерелятивистским задачам.

Благодарности. Автор благодарит профессоров Р. А. Минлоса и Д. П. Желобенко за внимание и обсуждение результатов этой работы.

\section{Список литературы}

[1] Н. Н. Боголюбов, Д. В. Ширков. Введение в теорию квантованных полей. М.: Наука, 1958.

[2] И. М. Гельфанд, Г. Е. Шилов. Обобщенные функции. Вып. 1. М.: Физматгиз, 1958.

[3] И. М. Гельфанд, Н. Я. Виленкин. Обобщенные функции. Вып. 4. М.: Физматгиз, 1961.

[4] Г. Е. Шилов. Математический анализ, второй специальный курс. М.: Наука, 1965.

Поступила в редакцию $22 . \mathrm{XI} .2004$ г., после доработки 28.II.2005 г. 